\title{
Glaucoma Surgery with Soaked Sponges with Mitomycin C vs Sub-Tenon Injection: Short-term Outcomes
}

\author{
Maria EV Guimarães ${ }^{1}$, Bernardo de Pádua Soares Bezerra², Frederico de Miranda Cordeiro ${ }^{3}$, Christiano HS Carvalho ${ }^{4}$, \\ Daniella N Danif ${ }^{5}$, Tiago S Prata ${ }^{6}$, Syril K Dorairaj ${ }^{7}$, Fábio N Kanadani ${ }^{8}$
}

\begin{abstract}
Aim: The aim of this study is to compare the outcomes, success rates, complications, and number of interventions of the trabeculectomy surgery with mitomycin $\mathrm{C}(\mathrm{MMC})$ in two different techniques: conventional soaked sponges and sub-Tenon injection.

Materials and methods: An observational retrospective study was performed with 79 eyes of 64 participants who underwent a glaucoma surgery with MMC. The conventional sponge soaked with MMC $0.03 \%$ for 3 minutes over the scleral flap (group I) and the subtenon injection of $0.1 \mathrm{~mL}$ of MMC $0.03 \%$ (group II) techniques were used to deliver the MMC. The data collected were patient demographics, preoperative intraocular pressure (IOP), central corneal thickness (CCT), number of pre- and postoperative ocular hypotensive drugs, postoperative complications, number of interventions, and need of further glaucoma surgery.

Results: Seventy-nine eyes were divided into two: 39 eyes (49.37\%) in group I and 40 eyes (50.63\%) in group II. Trabeculectomy was performed in $53.17 \%$ and phacotrabeculectomy in $46.83 \%$. The IOP decreased from $18.0 \pm 11.0 \mathrm{~mm} \mathrm{Hg}$ at the baseline to $10.0 \pm 3.0 \mathrm{~mm} \mathrm{Hg}$ at the last visit in group I and, in group II, from $16.0 \pm 10.0-13.0 \pm 6.0 \mathrm{~mm} \mathrm{Hg}$ (median \pm ID), $p<0.001$. There were no significant differences between the groups regarding demographics, median follow-up, IOP in baseline, CCT, drugs reduction, success criteria adopted, postsurgical interventions, surgical complications, and need for new procedures $(p>0.05)$.

Conclusion: Sub-Tenon injection of MMC is a safe and as effective as the conventional soaked sponge method in trabeculectomies. This method reduces surgical time and sponge-related risks with equivalent surgical efficacy.

Clinical significance: As trabeculectomy is still the most common surgical procedure to reduce IOP and scar formation is one of the main causes of surgical failure, it is important to develop surgical techniques to improve its outcomes.

Keywords: Glaucoma, Mitomycin C, Outcome, Surgery, Trabeculectomy.

Journal of Current Glaucoma Practice (2019): 10.5005/jp-journals-10078-1254
\end{abstract}

\section{INTRODUCTION}

Trabeculectomy is the most common surgical procedure performed to effectively reduce IOP to prevent further glaucomatous optic nerve damage.

Despite its pitfalls, it is still a first-line surgery with a favorable cost/benefit ratio. ${ }^{2,3}$ Even with advances in topical treatment, new drug delivery methods and new surgical techniques including glaucoma drainage devices and minimally invasive glaucoma surgery, the conventional trabeculectomy still holds its place in the treatment arsenal against the silent thief of sight. Therefore, it seems realistic to try and modify or revise the classical approach to achieve higher success rates and reduced complication levels.

The most common cause of failure of glaucoma filtering procedures is scar formation at the surgical site. ${ }^{4}$ Since their introduction in 1990, antifibrotic agents, such as MMC and 5-fluorouracil (5-FU), have increased the long-term success rates of filtering surgery. 5,6 The method of the antimetabolite application during trabeculectomy surgery may change the morphology of the drainage bleb and its long-term survival. ${ }^{7}$

The conventional technique of MMC use involves direct application of the soaked sponges either before ${ }^{8,9}$ or after $^{10,11}$ the scleral flap dissection. Drawbacks to MMC application with sponge include longer duration of the procedure, inability, and the risk of losing or retaining sponge fragments under the conjunctiva. ${ }^{12}$

Lee et al. ${ }^{13}$ described a novel technique of MMC application in which it is injected into Tenon's layer prior to scleral flap formation, giving it a diffuse area of action. The study concluded that after
${ }^{1,3-5}$ Department of Ophthalmology, Instituto de Olhos Ciências Médicas, Belo Horizonte, Minas Gerais, Brazil

${ }^{2}$ Department of Ophthalmology, Instituto de Olhos Ciências Médicas, Belo Horizonte, Minas Gerais, Brazil; Royal Victorian Eye and Ear Hospital, Melbourne, Australia

${ }^{6}$ Department of Ophthalmology, Mayo Clinic, Jacksonville, Florida, USA; Department of Ophthalmology and Visual Science, Federal University of São Paulo, São Paulo, Brazil; Glaucoma Unit, Hospital Medicina dos Olhos, Osasco, São Paulo, Brazil; Department of Ophthalmology, Glaucoma Service, Hospital Oftalmológico de Sorocaba-BOS, Sorocaba, São Paulo, Brazil

${ }^{7}$ Department of Ophthalmology, Mayo Clinic, Jacksonville, Florida, USA

${ }^{8}$ Department of Ophthalmology, Instituto de Olhos Ciências Médicas, Belo Horizonte, Minas Gerais, Brazil; Department of Ophthalmology, Mayo Clinic, Jacksonville, Florida, USA

Corresponding Author: Maria EV Guimarães, Department of Ophthalmology, Instituto de Olhos Ciências Médicas, Belo Horizonte, Minas Gerais, Brazil, Phone: +55 31 991098034, e-mail:mevguimaraes@ hotmail.com

How to cite this article: Guimarães MEV, de Pádua Soares Bezerra B, et al. Glaucoma Surgery with Soaked Sponges with Mitomycin C vs Sub-Tenon Injection: Short-term Outcomes. J Curr Glaucoma Pract 2019;13(2):50-54.

Source of support: Nil

Conflict of interest: None

(c) The Author(s). 2019 Open Access This article is distributed under the terms of the Creative Commons Attribution 4.0 International License (https://creativecommons. org/licenses/by-nc/4.0/), which permits unrestricted use, distribution, and non-commercial reproduction in any medium, provided you give appropriate credit to the original author(s) and the source, provide a link to the Creative Commons license, and indicate if changes were made. The Creative Commons Public Domain Dedication waiver (http://creativecommons.org/publicdomain/zero/1.0/) applies to the data made available in this article, unless otherwise stated. 
12 months follow-up, the technique was efficacious and favorably comparable to others in the literature.

In this study, we retrospectively compared the outcomes, success, complications rates, and number of further intervention of two different MMC application techniques-conventional soaked sponges and sub-Tenon injection, during trabeculectomies and phacotrabeculectomies.

\section{Materials and Methods}

This single center, observational, retrospective study was carried out at the Instituto de Olhos Ciências Médicas, Belo Horizonte, Minas Gerais, Brazil.

A chart review of patients who underwent an antiglaucoma surgical procedure, either trabeculectomy or phacotrabeculectomy, was performed. All surgeries with adjuvant use of MMC were performed by a single experienced surgeon (FK) from 2014 to 2016.

Patient informed consent was not required due to the observational and retrospective nature of the study where all information came from medical charts' review, with no intervention or deliberate modification of biologic, physiologic, psychological, or social variables. All patients with a follow-up period less than 6 months were excluded from the study.

Collected data included patient demographics, preoperative IOP, CCT, number of pre- and postoperative ocular hypotensive drugs, postoperative complications and interventions, and number of further glaucoma surgery. IOP was measured through every visit and in the latest follow-up visit using the Goldmann applanation tonometry.

The postoperative complications considered were as follows: malignant glaucoma, hypotonia (IOP $<6 \mathrm{~mm} \mathrm{Hg}$ ), hypotonic maculopathy, bleb leaking, blebitis, hyphema, choroidal detachment, iris obstructing the trabeculectomy ostium, cataract, endophthalmitis, cystoid macular edema, and hyperfiltrating bleb.

The further surgeries considered were the re-trabeculectomy and the drainage implant (tube).

The two success criteria were considered as follows: (la) IOP less than or equal to $18 \mathrm{~mm} \mathrm{Hg}$ without medication (completed success) and (Ib) with medication (qualificated success) and (Ila) IOP less than or equal to $15 \mathrm{~mm} \mathrm{Hg}$ without medication (completed success) and (IIb) with medication (qualificated success).
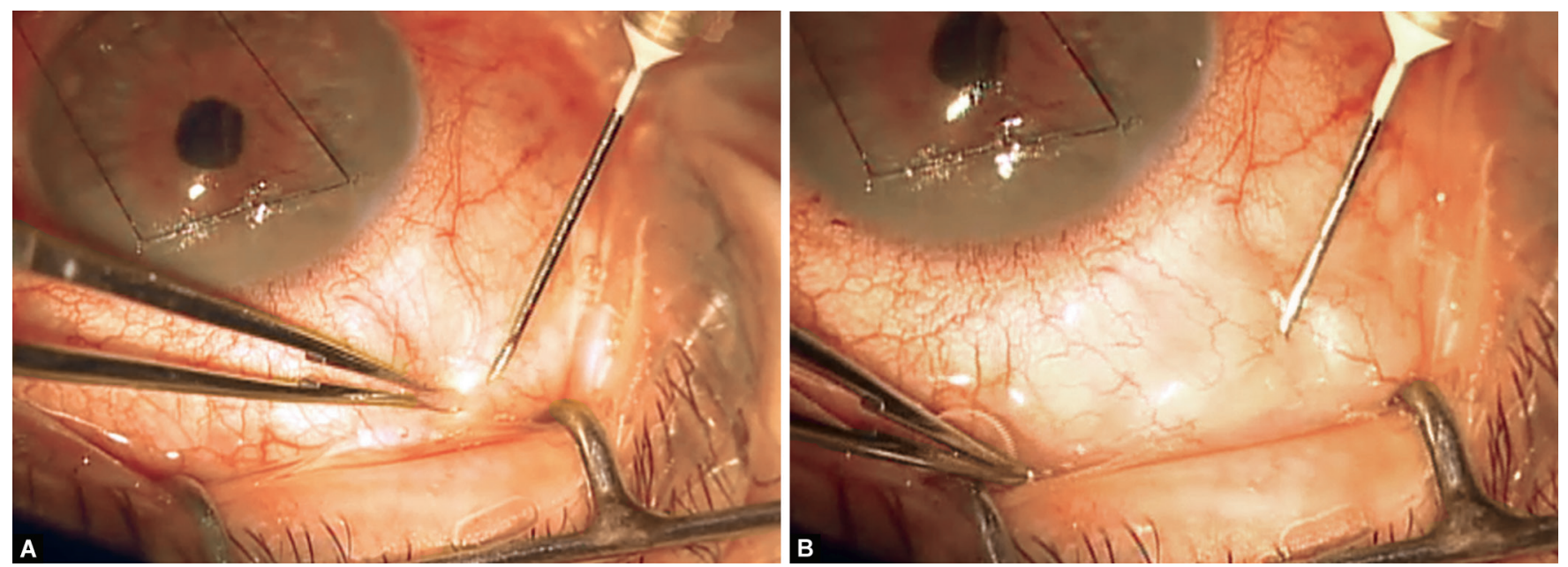

\section{Surgical Technique}

Trabeculectomy surgery was performed under peribulbar anesthesia. An 8-0 silk corneal stay was used to retract the globe inferiorly. In the conventional soaked sponges group (group I), an initial standard fornix-based conjunctival incision was created. Three $0.03 \%$ MMC-soaked microswabs were placed subconjunctivally for 3 minutes with subsequent no irrigation. The surgery was then continued per protocol. In the intra-Tenon injection of the MMC group (group II), with the eye displaced inferiorly, a 30-gauge needle was passed through conjunctiva and advanced laterally in Tenon's along the superior aspect of the globe. MMC $(0.1 \mathrm{~mL}$ at $0.03 \%$ ) was injected slowly (Fig. 1), while resting a cannula anteriorly and posteriorly to prevent spread over the future site of the conjunctival wound. Right after the subtenon injection, the perilimbal conjunctiva was dissected and the conventional steps of trabeculectomy procedure were followed. Postoperative treatment was standard. Topical antibiotic drops (moxifloxacin $5.45 \mathrm{mg} / \mathrm{mL}$ ) were used 4 times a day for 1 week and topical steroid (prednisolone $1 \%)$ drops were used frequently for the early postoperative period and gradually tapered over the following 8 weeks.

\section{Statistical Analysis}

The categorical variables were presented as counts and percentages, and the numerical values as mean \pm standard deviation (SD), if they had a normal distribution (by the Shapiro-Wilk test), or median \pm interquartile distance (ID), in case they were not normally distributed. The comparison of two means between the groups was performed via Student's $t$ tests or Wilcoxon Mann-Whitney for independent samples. The association of categorical variables was assessed using Fisher's exact tests or Chi-square of independence. Means longitudinal comparisons were made by the paired Wilcoxon test. Kaplan-Meier curves were constructed to evaluate patient survival (indicate the event and the initial and final times), compared using the log-rank test. The analysis was developed in program $R$ version 3.3 .2 and a significance level of $5 \%$ was adopted.

\section{Results}

The study included 79 eyes of 64 participants who underwent glaucoma surgery. In 39 eyes (49.37\%), a sponge with subconjunctival MMC (group I) was used, while MMC injection (group II) was performed in 40 eyes (50.63\%). The median follow-up period was

Figs $1 \mathrm{~A}$ and B: Intra-Tenon injection of MMC $0.1 \mathrm{~mL}$ at $0.03 \%$ 
Table 1: Demographic and ocular characteristics of study patients

\begin{tabular}{|c|c|c|c|}
\hline Variables & Group I $(n=39)$ & Group II $(n=40)$ & $p$ value \\
\hline Gender female & $21(63.64 \%)$ & $20(64.52 \%)$ & $1.000^{Q}$ \\
\hline Age (years) ${ }^{1}$ & $68.0 \pm 16.0$ & $73.0 \pm 14.0$ & $0.224^{\mathrm{W}}$ \\
\hline $\begin{array}{l}\text { Baseline number of } \\
\text { medication }{ }^{1}\end{array}$ & $2.0 \pm 1.0$ & $2.0 \pm 1.0$ & $0.612^{\mathrm{W}}$ \\
\hline Baseline IOP $(\mathrm{mm} \mathrm{Hg})^{1}$ & $18.0 \pm 11.0$ & $16.0 \pm 10.0$ & $0.664^{\mathrm{W}}$ \\
\hline $\mathrm{CCT}(\mu \mathrm{m})^{2}$ & $516.9 \pm 38.6$ & $507.9 \pm 34.7$ & $0.325^{\top}$ \\
\hline
\end{tabular}

${ }^{1}$ Data presented as median \pm ID

${ }^{2}$ Mean \pm SD

The $p$ values refer to the test: ${ }^{Q}$ qui-square of independence

WWilcoxon Mann-Whitney

${ }^{T} t$-Student for comparison of independent samples

$12.0 \pm 0.0$ and $12.0 \pm 3.0$ months in groups I and II, respectively $(p=0.058)$. Clinical and demographic characteristics of the sample are shown in Table 1. In relation to the surgical technique, trabeculectomy was performed in $53.17 \%$ of eyes and combined phacotrabeculectomy in $46.83 \%$. There was no significant difference between groups I and II with regard to gender, age, number of ocular hypotensive medications, baseline IOP, and CCT ( $p>0.05)$.

In group I, IOP decreased from $18.0 \pm 11.0 \mathrm{~mm} \mathrm{Hg}$ at the baseline to $10.0 \pm 3.0 \mathrm{~mm} \mathrm{Hg}(p<0.001)$ at the last follow up visit and, in group II, from $16.0 \pm 10.0 \mathrm{~mm} \mathrm{Hg}$ to $13.0 \pm 6.0 \mathrm{~mm} \mathrm{Hg}(p<0.001)$. The percentage of reduction was $47.37 \pm 26.67 \%$ in group I and $33.33 \pm 31.25 \%$ in group II $(p=0.030)$.

The number of ocular hypontensive medications reduction was $2.0 \pm 1.0$ drugs in group II and $2.0 \pm 1.0$ in group II $(p=0.680)$.

On the survival analysis at 10 months postoperatively, in group I, the success rate for criterion la (IOP less than or equal to $18 \mathrm{~mm} \mathrm{Hg}$ without medication) was 0.763 and $\mathrm{Ib}$ (IOP less than or equal to $18 \mathrm{~mm} \mathrm{Hg}$ with medication) was 0.974. Considering criterion lla (IOP less than or equal to $15 \mathrm{~mm} \mathrm{Hg}$ without medication), the success rate was 0.763 and IIb (IOP less than or equal to $15 \mathrm{~mm} \mathrm{Hg}$ with medication) was 0.974 . Curiously, the survival analysis demonstrates the same findings for criteria la and Ila such as Ib and IIb in group I.

In the group II, the success rate for criterion la was 0.723 and, for the criterion Ib, it was 0.875 . Considering criterion II, the complete success rate (Ila) was 0.693 and, for the qualified one (Ilb), it was $0.824(p>0.05)$ (Figs 2 and 3 ).

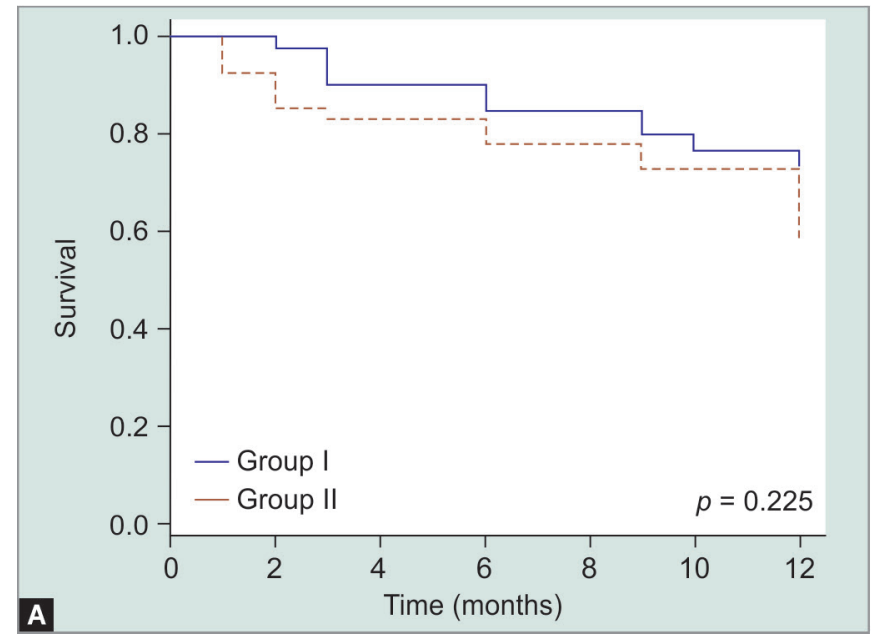

Figs 2A and B: Survival curves criterion I; (A) Complete; (B) Qualified
Considering surgical complications, 20 eyes in total had 26 postoperative complications, 14 in group I (35.9\%) and 12 in group II (30.0\%). The most common complication was hyphema, 6 in total $(7.6 \%)$, followed by 4 choroidal detachments $(5.1 \%)$, equally distributed in both groups. More complications were described, such as malignant glaucoma ( 2 cases within the first week in group II, $2.5 \%$ ), blebitis (one case at the 6 th week and other case at the 6th month in group I, 2.5\%), and hypotony (one case at the 6th month in group I, 1.3\%). These two malignant glaucoma cases occurred in both eyes of the same patient. There were no significant differences in surgical complications $(p=0.750)$ between groups I and II.

Another glaucoma surgery was performed in four patients (10\%) only in group II (tube implantation) $(p=0.116)$.

In group I, the interventions performed were 10 suture lysis (25.6\%) and 4 bleb needling (10.3\%). In group II, 14 suture lysis (35.0\%) were performed, and 8 bleb needling (20.0\%) with no significant differences in suture lysis $(p=0.510)$ and needling $(p=0.348)$.

\section{Discussion}

Trabeculectomy is still the most common and effective surgical procedure for lowering IOP in glaucoma. Since its first description, several technical modifications have been applied, like the use of antimetabolites, releasable sutures, and new surgical instruments. $^{14}$

The Moorfields Safe Surgery System (MSSS) increased safety and efficacy of the procedure. ${ }^{15,16}$ This surgical technique has, a fornix base approach, large Tenon's dissection and use of antimetabolite area, careful construction of the scleral flap, use of anterior chamber maintainer, and a thigh conjunctival closure.

The MMC use has changed over the years, mainly in its concentration and the way of application. Initially, different MMC concentrations were soaked to sponges and placed under the scleral flap at different times, generally from 1 to 5 minutes. Concerns and risks regarding the use of sponges include the possibility of sponge fragmentation, retention, excessive conjunctiva manipulation, and a longer surgical time.

Lee et al. $^{13}$ first reported intra-Tenon injection of MMC for trabeculectomies and phaco-trabeculectomies on a total of 108 cases that received different concentrations of the drug, from

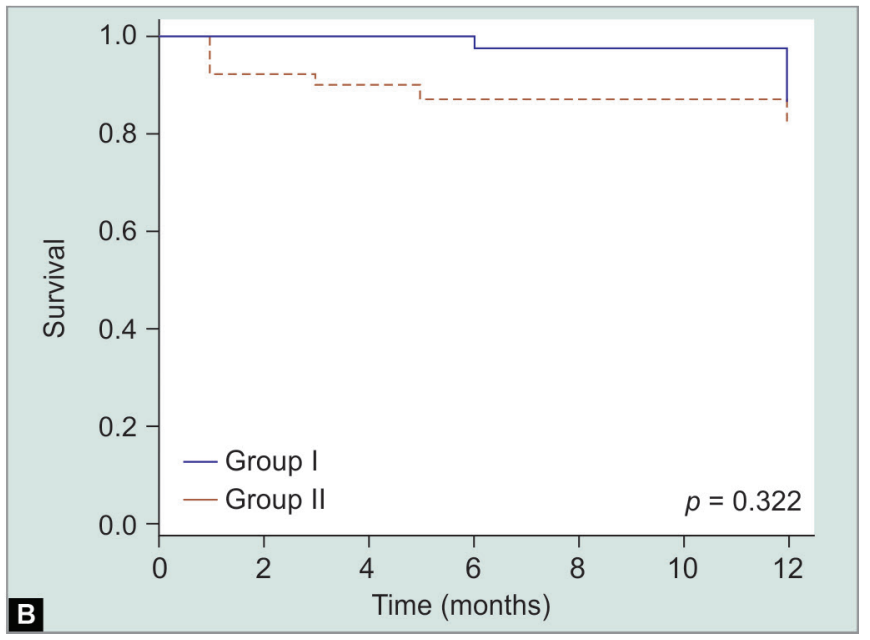




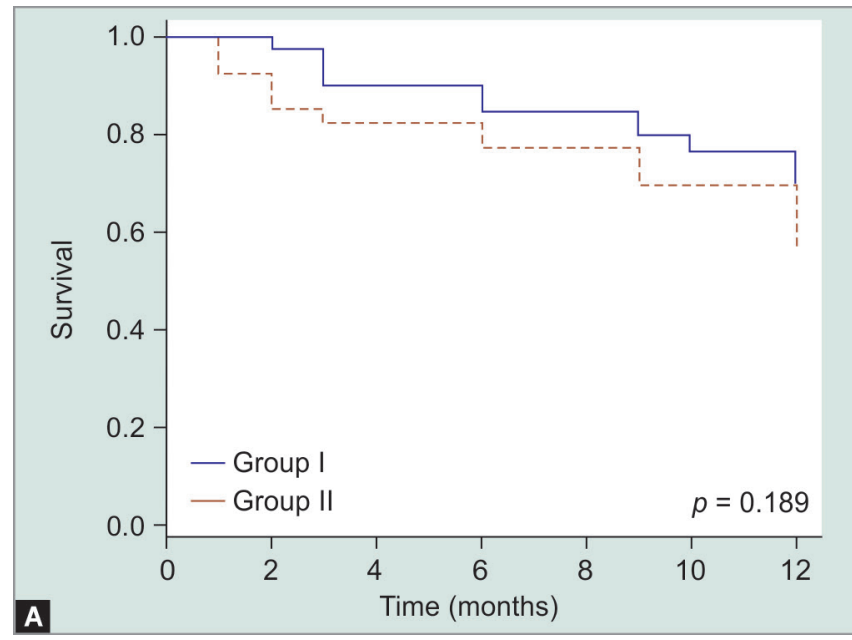

Figs 3 A and B: Survival curves criterion II; (A) Complete; (B) Qualified

0.2 to $0.5 \mathrm{mg} / \mathrm{mL}$ just before the surgery. In a follow-up study using the same technique, Lim et al. ${ }^{17}$ compared 125 trabeculectomies performed with MMC $0.1 \mathrm{~mL}$ of 0.05 or $0.1 \mathrm{mg} / \mathrm{mL}$ in sponges and 57 with intra-Tenon injection. Although they did not find any difference in efficacy between the groups regarding IOP reduction, a lower number of glaucoma eye drops and a number of 5 -fluorouracil injections were found in the intra-Tenon injection group.

A randomized clinical trial conducted by Pakravan et al. ${ }^{18}$ reported a diffuse, less vascularized, and shallower bleb when used subtenon injection of $0.1 \mathrm{~mL}$ of $0.01 \% \mathrm{MMC}$, suggesting a favorable bleb morphology with this technique.

In this study, was used a fixed MMC concentration of $0.03 \%$ in both techniques, different from Pakravan et al. ${ }^{18}$ which utilized the subtenon injection of MMC with a concentration of $0.01 \%$ and soaked sponge of $0.02 \%$. Lee et al. ${ }^{13}$ used MMC injection ranging from 0.02 to $0.05 \%$ depending on the risk of preoperative bleb failure.

Sub-Tenon injection of MMC during trabeculectomy is a safe, effective, and faster alternative to the conventional sponge method. The procedure is direct and prevents the preparation of sponge materials and complications related to its use as risk of loss or retention of sponge fragments under the conjunctiva, for example. In this technique, the surgeon can objectively determine the exact dose of MMC delivered to the tissue and adjust it for each patient accordingly to the risk of bleb failure. ${ }^{18}$

In our study, there was no statistically significant difference in surgical outcomes, success rates, complications, and need for further intervention between the studied groups. It is important to highlight some limitations in the present study: first, a relatively short follow-up period (11.46 \pm 1.17 months in group I and $10.65 \pm$ 1.79 months in group II); second, some eyes were excluded because of the lack of information and retrospective design of the study; and, finally, the surgical time was not recorded.

\section{Conclusion and Clinical Significance}

In conclusion, sub-Tenon injection of MMC is a safe, effective, and faster alternative to the conventional soaked sponge method. Further studies with a longer follow-up period are needed to evaluate long-term side effects of sub-Tenon MMC injection.

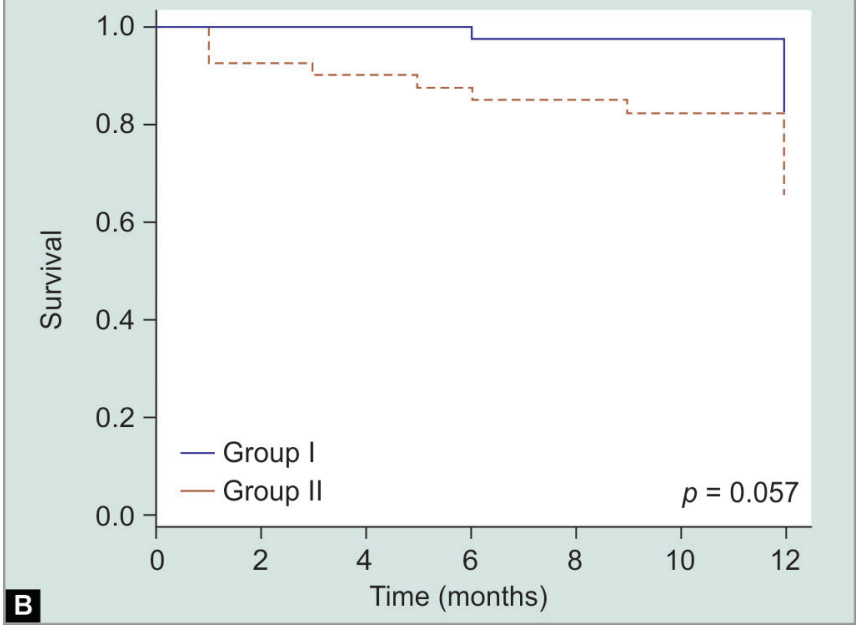

\section{ACKNOWLEDGMENT}

All authors have no financial disclosures or support in this work.

\section{Author Contributions}

All authors contributed equally to this work.

\section{References}

1. Coleman AL. Advances in glaucoma treatment and management: surgery. Invest Ophthalmol Vis Sci 2012;53(5):2491-2494. DOI: 10.1167/ iovs.12-9483I.

2. Gedde SJ, Herndon LW, et al. Surgical complications in the Tube vs Trabeculectomy Study during the first year of follow-up. Am J Ophthalmol 2007;143(1):23-31. DOI: 10.1016/j.ajo.2006. 07.022.

3. Edmunds B, Thompson JR, et al. The National Survey of Trabeculectomy. II. Variations in operative technique and outcome. Eye (Lond) 2001;15:441-448. DOI: 10.1038/eye.2001.152.

4. Skuta GL, Parrish II RK. Wound healing in glaucoma filtering surgery. Surv Ophthalmol 1987;32(3):149-170. DOI: 10.1016/00396257(87)90091-9.

5. Joshi AB, Parrish RK, et al. 2002 survey of the American Glaucoma Society: practice preferences for glaucoma surgery and antifibrotic use. J Glaucoma 2005;14(2):172-174. DOI: 10.1097/01. ijg.0000151684.12033.4d.

6. Wilkins $M$, Indar A, et al. Intra-operative mitomycin C for glaucoma surgery. Cochrane Database Syst Rev 2001(1):CD002897. DOI: 10.1002/14651858.CD002897.

7. You YA, GuYS, et al. Long-term effects of simultaneous subconjunctival and subscleral Mitomycin-C application in repeat trabeculectomy. J Glaucoma 2002;11(2):110-118. DOI: 10.1097/00061198-20020400000006.

8. Falck FY Jr, Skuta GL, et al. Mitomycin vs 5-fluoruracil anti-metabolite therapy for glaucoma filtration surgery. Semin Ophthalmol 1992;7: 97-109. DOI: 10.3109/08820539209065098.

9. Sisto $D$, Vetrugno $M$, et al. The role of antimetabolites in filtration surgery for neovascular glaucoma: intermediate-term follow-up. Acta Ophthalmol Scand 2007;85(3):267-271. DOI: 10.1111/j.16000420.2006.00810.x.

10. Beatty S, Potamitis T, et al. Trabeculectomy augmented with Mitomycin-C application under thescleral flaps. Br J Ophthalmol 1998;82(4):397-403. DOI: 10.1136/bjo.82.4.397. 
11. Palmer SS. Mitomycin as adjunct chemotherapy with trabeculectomy. Ophthalmology 1991;98(3):317-321. DOI: 10.1016/S01616420(91)32293-0.

12. Shin $\mathrm{DH}$, Tsai $\mathrm{CS}$, et al. Retained cellulose sponge after trabeculectomy with adjunctive subconjunctival mitomycin-C. Am J Ophthalmol 1994;118(1):111-112. DOI: 10.1016/S0002-9394(14) 72853-3.

13. Lee E, Doyle E, et al. Trabeculectomy surgery augmented with intra-Tenon injection of mitomycin C. Acta Ophthalmol 2008;86(8): 866-870. DOI: 10.1111/j.1755-3768.2007.01147.x.

14. Sawchyn AK, Slabaugh MA. Innovations and adaptations in trabeculectomy. Curr Opin Ophthalmol 2016;27(2):158-163. DOI: 10.1097/ICU.0000000000000236.
15. Khaw PT, Chiang M, et al. Enhanced trabeculectomy: the Moorfields SaferSurgery System. Dev Ophthalmol 2012;50:1-28. DOI: 10.1159/000334776.

16. Khaw PT. Advances in glaucoma surgery: evolution of antimetabolite adjunctive therapy. J Glaucoma 2001;10(5):S81-S84. DOI: 10.1097/00061198-200110001-00029.

17. Lim MC, Tong MG, et al. A comparison of trabeculectomy surgery outcomes with mitomycin-C applied by intra-tenon injection vs sponge method. American Glaucoma Society 23rd Annual Meeting 2013.

18. Pakravan $\mathrm{M}$, Esfandiari $\mathrm{H}$, et al. Mitomycin $\mathrm{C}$-augmented trabeculectomy: subtenon injection vs soaked sponges: a randomised clinical trial. Br J Ophthalmol 2017;101(9):1275-1280. DOI: 10.1136/ bjophthalmol-2016-309671. 